\title{
DIFERENCIAS SALARIALES Y DESAJUSTE EDUCATIVO EN COLOMBIA
}

\author{
HÉCTOR ALBERTO BOTELLO-PEÑALOZA Y \\ ISAAC GUERRERO RINCÓN \\ Universidad Industrial de Santander, Bucaramanga, Colombia
}

\section{RESU MEN}

Este artículo analiza el desajuste educativo en el primer empleo de los graduados de educación terciaria en Colombia y sus efectos en los ingresos laborales. Con base en los microdatos de dos encuestas (la encuesta longitudinal de protección social y la del Ministerio de Educación), se utilizó la metodología objetiva y subjetiva para calcular el desajuste en el año 2012. Los resultados muestran que entre un 22 y $35 \%$ de los egresados se encuentran sobre cualificados a la vez que ganan hasta un $4 \%$ menos que los correctamente educados. Ante esto, se debe impulsar la continua revisión de los contenidos teóricos en las asignaturas de la educación terciaria para que se adapten a las competencias reales a usar en el mercado laboral.

Palabras claves: empleo, capital humano, Colombia, productividad, egresados

Clasificaciones JEL: I21, J01, J24, C51, I23

Esta investigación hace parte del proyecto sobre Capital social y Desarrollo del grupo de Grupo de Investigación sobre Desarrollo Regional y Ordenamiento Territorial, reconocido por Colciencias en la categoría B. Correo electrónico: Héctor Alberto Botello-Peñaloza, hectoralbertobotello@gmail.com. Recibido: 1 de junio de 2019; aceptado: 29 de noviembre de 2019.

Econamá \& Ragón Vd. 13, Nb. 2, (Gartagena, dienbre 2019), pp 177- 191 


\section{ABST RACT}

\section{Educational mismatch of college graduates in Colombia}

We study the educational mismatch in the first employment of college graduates in Colombia and its wage effects, using microdata obtained from two surveys (the Longitudinal Social Protection Survey and the Ministry of Education Survey). We used the objective and subjective methodology to calculate the educational mismatch in 2012. The results show that between 22 and $35 \%$ of the graduates are overeducated in their jobs while earning up to $4 \%$ less than the adequately educated workers. These results suggest the need to foster a constant update of the theoretical contents in tertiary-education courses, in order to adjust them to fit the competence expectations of the labor market.

Key words: labor, human capital, Colombia, productivity, graduates

JEL Classifications: I21, J01, J24, C51, I23

\section{INTRODUCCIÓN}

La oferta laboral en el mercado de trabajo está constituida por un conjunto de recursos humanos altamente heterogéneos, a causa principalmente de las competencias y cualificaciones específicas que desarrolla cada trabajador. Estos conocimientos y habilidades han sido adquiridos, en su mayoría, por medio del capital humano formal, expresado de manera específica en los grados de educación escolar primaria, secundaria y terciaria. Para promover la mayor cualificación y especialización productiva, los gobiernos de los países han realizado grandes inversiones en programas sociales de educación y en el mejoramiento de la calidad universitaria.

No obstante, es posible que todas estas inversiones estén mal orientadas, si las aptitudes desarrolladas en el ámbito escolar no coinciden con las requeridas por las empresas. A este fenómeno se le conoce como desajuste educativo, y se produce cuando se subvalora o sobrevalora la dotación de capital humano que posee el trabajador dentro de su ocupación (Freeman, 1975). Para los recién graduados de educación terciaria, las posibilidades de acceder a un empleo son difíciles dado que los empleadores, aun conociendo los contenidos de las 
carreras, consideran que la universidad sólo transmite conocimientos teóricos, que no contribuye al desempeño dentro de trabajos tales como el pensamiento creativo y la capacidad para resolver problemas y la motivación (Carnevale, 1990; Velasco, 2004).

En este sentido, es de interés científico estudiar si los egresados pueden colocarse en el mercado laboral donde puedan poner en práctica los conocimientos aprendidos o, por el contrario, si no existe conexión alguna entre estos conocimientos y el sector productivo. Esta pregunta de investigación se ve motivada por dos hechos. El primero es que el estudio de la educación terciaria es importante dado que su retorno salarial es el más significativo dentro de los diferentes niveles de educación en Colombia (Chávez y Arias, 2002). El segundo es que la población ocupada en Colombia ha venido incrementando sus niveles educativos promedio en tiempos recientes. Según las encuestas de hogares del DANE de 2009, las personas con educación inferior a la media representaban el 54,9\% de los ocupados totales mientras que actualmente, a 2017, este mismo porcentaje se ha reducido a 46,4\%. Al mismo tiempo, ha aumentado la participación de personas con título de bachillerato y educación superior, de $27,7 \%$ a $31,9 \%$ y de $17,4 \%$ a $21,7 \%$ en el mismo periodo. Por 10 tanto, existen preocupaciones sobre el hecho de que este incremento de las cualificaciones no se vea reflejado en las necesidades de las empresas.

En consecuencia, el articulo contribuye a la literatura existente explorando el grado de desajuste educativo del primer empleo de los egresados de educación terciaria en Colombia.

Para lograr lo anterior, el artículo se compone de cuatro secciones aparte de esta. En la segunda, se presenta las causas del desajuste educativo junto con la literatura científica reciente que trata este tema. Posteriormente se exponen las fuentes de información junto con el modelo implementado para cuantificar las consecuencias del desajuste educativo en los salarios de los egresados. Finalmente, en la cuarta sección se muestran los resultados del estudio y las conclusiones.

Existen diferentes puntos de vista del origen del desajuste educativo de modo que resumiremos las principales teorías asociadas con el tema. En primer lugar, para la teoría neoclásica, el desajuste educativo es un problema friccional temporal del mercado de trabajo (Johnson, 1978). Según Sicherman (1991) el número de puestos calificados es limitado en cierto periodo del tiempo, por lo tanto, no todos los trabajadores poseedores del nivel de capital humano adecuado son ocupados. Sin embargo, con el tiempo el mercado generará los puestos disponibles a medida que la oferta y la demanda laboral se ajusten, por 
lo que concluye que el fenómeno es de tipo temporal. Para algunos autores, como Rosen (1972) y Sicherman (1991), el desajuste educativo también resulta un fenómeno temporal, pero por causa de la movilidad laboral. En ocasiones, los trabajadores ejecutan temporalmente trabajos para los que están sobreeducados para adquirir la experiencia necesaria para acceder a trabajos más calificados. Desde los modelos de señalización, el desajuste educativo es un proceso estructural, que se deriva del rápido aumento en el número de personas que invierten en educación, haciendo que las empresas sean incapaces de absorberlos para sus necesidades productivas (Spence, 1973).

En este orden de ideas, para el cálculo empírico del desajuste educativo han surgido tres metodologías principales:

1. El método estadístico: se parte del promedio en los años de educación dentro de un grupo ocupacional a considerar, con rango de más o menos una desviación estándar. Un individuo está sobre-educado si tiene más años de educación que la media más la desviación estándar del grupo de referencia y viceversa para los infra-educados (Botello, 2015). La dificultad principal de este método consiste en hallar cómo fijar el valor medio del nivel educativo (Dorn y Souza-Posa, 2005); por ejemplo, si en el grupo observado la sobreeducación es alta, se eleva artificialmente el valor de referencia. ${ }^{1}$ Pero incluso con este problema, Hartog (2000) asegura que el método estadístico es el más implementado, por su facilidad de cálculo y la disponibilidad de datos estadísticos. Sin embargo, Flisi et al. (2017), aseguran que aun cuando la literatura suela usar una desviación estándar, es posible estimar el desajuste con un valor más alto o más bajo en consideración a cómo se quiera validar la severidad del desajuste educativo. Por esto es necesario considerar con cautela este método.

2. El método subjetivo: consiste en indagar con los propios ocupados sobre cuáles credenciales consideran que son las apropiadas para efectuar las tareas de su puesto de trabajo. Estos datos se cruzan con sus actuales cualificaciones, indicando a continuación si se considera sobre-educado o no. Las apreciaciones subjetivas poseen errores de clasificación ya que el investigador no conoce cómo el encuestado fijó el nivel educativo necesario para el puesto (Mora, 2004; McGuiness, 2006).

\footnotetext{
${ }^{1}$ Groot y van den Brink (2000) evidencian la diferencia que puede haber entre un método y otro. En un análisis de 25 ocupaciones encontraron que el desajuste verdadero era del 26\%, mientras que la estimada con la media fue del 12\%. Según Kiker et al. (1997), Ng (2001) y Rubb y Quinn (2002), el valor referencial se debe tomar con base en la mediana de la distribución ocupacional para que no se vea afectado por valores repetidos o extremos.
} 
3. El método objetivo: requiere conocer cuáles son las competencias exigidas por las empresas y las poseídas por los trabajadores; el grado de incoherencia que haya entre los dos indicadores arroja el grado de desajuste educativo (Castillo, 2007). No obstante, resulta arduo conocer toda la información de cada uno de los puestos de trabajo dentro de las empresas y las competencias adquiridas por las personas.

4. Método de competencias: según Flisi et al. (2017) el desajuste educativo también puede ser medido por las competencias que manejan los trabajadores y su relación con las necesitadas por las empresas. Sin embargo, según los mismos autores el análisis de este punto de vista ha estado limitado por las encuestas.

A partir de la aplicación de los anteriores métodos dentro de la literatura empírica se encuentra que una mayor parte de los países suelen mostrar tasas permanentes de desajustes a raíz de las fricciones del mercado laboral (Hartog, 2000). Asimismo, aplicando modelos de capital humano, se ha evidenciado que las tasas de retorno de la educación de los sobre-educados es mayor que la de los infra-educados, pero menor en comparación con la de los adecuadamente educados (Mora, 2004).

Dentro de los trabajos aplicados a la población de egresados universitarios, Velasco (2004) estima por medio de preguntas subjetivas dirigidas a los egresados de la Universidad de Granada (España) en 1996, que el 37\% se encuentran infra-educados en sus trabajos contra un $11 \%$ que están sobre-educados. Además hallaron que las carreras Económicas y Empresariales, de Enfermería, y de Ciencias son títulos que han resultado significativamente favorables para el ajuste educativo. En otro trabajo, López (2008) investiga el desajuste educativo de los jóvenes en España con la Encuesta de Población Activa de ese país. Según los resultados el 55,5\% de los jóvenes se encuentra sobre-educado en su primer empleo, existiendo ciertas diferencias por género, de forma que el porcentaje dentro de las mujeres alcanza el 57,8\%, mientras que en el caso de los varones la cifra se reduce al 53,9\%.

En este orden de ideas, en aras de contribuir a la literatura internacional mostrando la experiencia de este fenómeno en Colombia, este trabajo estudia las causas y efectos del desajuste educativo para los egresados universitarios en su primer empleo, entendido como la diferencia por exceso o defecto, entre el nivel educativo alcanzado por el individuo y el exigido por el puesto de trabajo en el cual se desempeña. A continuación, se describe la metodología implementada. 


\section{METODOLOGÍA}

\section{A. Fuente de datos}

El análisis se basa en los datos estadísticos provenientes de la encuesta de egresados del Ministerio de Educación de Colombia hecha en 2014 y de la Encuesta Longitudinal de Protección Social (ELPS) provista por el Departamento Administrativo Nacional de Estadística (DANE) para 2012. ${ }^{2}$ De estas bases de datos se extrajeron cuatro grupos:

- Los egresados universitarios de la encuesta del Ministerio de Educación, que declaran que el empleo en el que están ocupados es su primera experiencia laboral. Después de la depuración, la población de la primera encuesta fue de 681 personas, ajustada por factores de expansión a 657 mil. - Los egresados universitarios de la ELPS que declararan que el empleo en el que están activos en su primera experiencia laboral. Esta muestra fue de 1.576 personas y, al aplicar los factores de expansión, corresponde a 1,5 millones de personas.

- Los trabajadores de la ELPS con educación terciaria que están en el mercado laboral en su segundo o posterior empleo, fueron 1.907 personas, ajustados a 1,9 millones.

- Los ocupados de la ELPS con educación diferente a la terciaria. Sumaron 6.162 personas ampliada hasta seis millones a causa de los factores de expansión.

Estos grupos se tomaron como referencia para comparar el grado de desajuste que poseen los egresados. El Cuadro 1 presenta la distribución de las personas con educación terciaria que se encuentran en su primer empleo por sexo y nivel educativo.

\footnotetext{
${ }^{2} \mathrm{Si}$ bien la encuesta puede ser un poco antigua, consideramos que posee información valiosa acerca de la educación de las personas y su trayectoria en el mercado laboral, que son aspectos que pocas encuestas en Colombia poseen.
} 


\section{CUADRO 1}

Distribución de los encuestados con educación terciaria que tienen su primer empleo, por sexo y nivel educativo

\begin{tabular}{|c|c|c|c|c|}
\hline \multicolumn{4}{|c|}{ Encuesta protección social } & Encuesta del ministerio \\
\hline Nivel & Hombre & Mujer & Total & Total \\
\hline $\begin{array}{c}\text { Universi- } \\
\text { tario }\end{array}$ & $50,60 \%$ & $51,40 \%$ & $51,00 \%$ & $64,85 \%$ \\
\hline $\begin{array}{c}\text { Tecnoló- } \\
\text { gico }\end{array}$ & $11,90 \%$ & $9,60 \%$ & $10,70 \%$ & $22,87 \%$ \\
\hline Técnico & $37,50 \%$ & $39,00 \%$ & $38,30 \%$ & $12,28 \%$ \\
\hline Total & 303.434 & 353.848 & 657.282 & 1.010 \\
\hline
\end{tabular}

Fuente: Encuestas Longitudinal de Protección social Colombia 2012, DANE.

\section{B. Cálculo del desajuste educativo}

Para estimar el tipo del desajuste educativo para cada población se realizaron los siguientes procedimientos: con la población de egresados de la encuesta del Ministerio se usó el método subjetivo. Existe una pregunta que pide que el entrevistado mencione qué nivel educativo considera que se requiere para elaborar la función que está haciendo. Si la respuesta se encuentra por debajo del nivel que posea el individuo se consideró sobre-educado; si es el mismo, correctamente educado; y si está por encima, infra-educado.

Para las poblaciones extraídas de la ELPS se usó el método objetivo. Se calculó la media y la desviación estándar de agrupaciones de ocupados por cada rama de actividad, ocupación y departamento. A partir de allí se ubicó al trabajador en los diferentes niveles de desajuste comparando el nivel educativo con la media más/menos una desviación estándar.

Posteriormente, con el uso de la medida estadística, se observaron las condiciones salariales que caracterizan a los egresados universitarios mediante el modelo presentado a continuación. 


\section{El efecto del desajuste en los ingresos laborales: modelo y estimación}

El modelo de Mincer (1974) permite estimar cuál es el efecto de las características de los trabajadores en sus ingresos laborales, por tal razón, para este artículo se quiere investigar cómo afecta el nivel de desajuste los retornos en el mercado laboral de los egresados de educación terciaria. Solo se pudo usar la información de las personas provenientes de la ELPS dado que solo allí se encuentran datos asociados a los salarios. El modelo cuenta como variables independientes con los años de educación del trabajador, la experiencia laboral, su cuadrado, la rama de actividad, su ocupación, el lugar de trabajo, el tamaño de la empresa, y la tenencia de contrato, entre otros (Ecuación 1).

$$
\log (Y)=\beta_{0}+\beta_{1} \operatorname{Educ}+\beta_{2} \operatorname{Exp}+\beta_{3} \operatorname{Exp}_{2}+\beta_{4} S+\beta_{5} L+\epsilon
$$

Donde:

- Y es el ingreso laboral por hora del individuo

- Educ es el nivel educativo

- Exp son los años de experiencia laboral

- L corresponde al tipo de desajuste educativo del egresado

- S son otras variables características del trabajador

- $\varepsilon$ es el término de perturbación aleatoria que se distribuye según una Normal

Entre las otras variables del trabajador están el departamento donde habita el individuo, el tamaño de la empresa donde labora, su etnia, su estado civil, la rama de actividad en la que se desempeña, y su satisfacción laboral, entre otras.

Adicionalmente, se consideraron las opiniones de Esquivel (2007) y Rivera (2013), para quienes dentro de la estructura de los datos de las encuestas de hogares se encuentra un problema denominado el sesgo de selección. El problema surge dada la presencia mayoritaria de cierto grupo poblacional que perturba las deducciones sobre las variables analizadas hacia las características de dicho grupo. La corrección se hace mediante el método de Heckman (1979), el cual consiste en utilizar variables instrumentales que permitan obtener estimadores consistentes en presencia de variables endógenas. Para esto se calcula primero una ecuación (Ecuación 2) que estime la probabilidad de participación en el mercado laboral usando como variables independientes los factores que pueden incidir en la elección, tales como la edad, los hijos, la raza, entre otros. 


$$
P_{i}=\beta 0+z_{i} \emptyset+U_{i}
$$

En esta estimación pi es la probabilidad de participar en el mercado laboral, $z_{i}$ es un vector de variables independientes, $\varphi$ es un vector de coeficientes, $y$ $u$ son los errores. Posteriormente, la ecuación de participación se adiciona al modelo de Mincer anteriormente expuesto (Ecuación 3).

$$
\log (Y)=\beta_{0}+\beta_{1} S+\beta_{2} \operatorname{Exp}+\beta_{3} \operatorname{Exp}^{2}+\beta_{4} L+\lambda_{t i} \theta+\epsilon_{i}
$$

Después de la estimación se prueban las siguientes hipótesis encontradas en la literatura (Mora y Muro, 2008):

H1: Los sobre-educados poseen ingresos menores a quienes están adecuadamente educados (Verdugo y Verdugo, 1989) pero mayores a los ingresos de los infra-educados. No obstante, se ha evidenciado que existen retornos positivos a la sobre-educación por encima de los demás niveles (Cohn y Kahn, 1995).

$\mathrm{H} 2$ : Se encuentran diferencias significativas entre los retornos para los sobreeducados en las diferentes ocupaciones (Verdugo y Verdugo, 1988; Cohn y Kahn, 1995; y Kiker et al., 1997).

\section{RESULTADOS}

El Cuadro 2 expone las estadísticas relacionadas con la distribución de trabajadores en la base total de empleo: el grado de desajuste laboral subjetivo afectó al 35\% de los individuos con educación terciaria ocupados en su primer empleo, con el $22 \%$ sobre-educados y $11 \%$ infra-educados. Por medio del método objetivo se observó que el $58 \%$ de la población tuvo desajuste, siendo $57 \%$ de estos sobre cualificados y solo un 1\% infra cualificados.

Para el resto de la población con educación terciaria en Colombia (es decir, quienes ya están más allá de su primer empleo) el nivel de desajuste educativo se mantuvo similar (47\%), al igual que la sobre-educación (46\%) y la infra-educación $(1 \%)$. Es decir, la sobre-educación se reduce en cerca de 11 pp (de 57,8\% a $46,5 \%$ ) una vez los trabajadores con educación terciaria abandonan su primer trabajo. Esto sería un resultado favorable para validar la postura que afirma que la sobre-educación puede ser una decisión óptima de los trabajadores jóvenes en su primer empleo (Schierman y Galor (1990)), en aras de ganar experiencia y habilidades para sus futuros trabajos. 


\section{CUADRO 2}

Porcentaje de trabajadores por tipo de desajuste laboral y población estudiada, 2012

\begin{tabular}{|c|c|c|c|c|}
\hline \multirow{2}{*}{ Tipo de desajuste } & \multicolumn{2}{|c|}{$\begin{array}{c}\text { Primer empleo } \\
\text { educación terciaria }\end{array}$} & \multicolumn{2}{c|}{ Resto de la población } \\
\cline { 2 - 5 } & Subjetivo & Objetivo & Educación terciaria & Total \\
\hline Infraeducado & 13,18 & 0,23 & 1,81 & 16,46 \\
\hline Correctamente & 64,8 & 41,97 & 51,62 & 62,99 \\
\hline Sobreeducados & 22,02 & 57,8 & 46,57 & 20,55 \\
\hline
\end{tabular}

Fuente: cálculos de los autores con base en la ELPS y la Encuesta de egresados del ministerio de educación.

Esto quiere decir que desde el punto de vista de la población con educación terciaria ocupada en su primer empleo el fenómeno del desajuste educativo es menos intenso del que se puede calcular mediante los métodos objetivos, resultado contrario al alcanzado por Velasco (2004). Por niveles educativos, el Cuadro 1 enseña los niveles educativos y el desajuste educativo correspondiente por tipo de encuesta. Los resultados muestran semejanzas entre las metodologías aplicadas: desde el punto de vista subjetivo y objetivo el desajuste educativo afecta al 35\% de la población con educación terciaria. Desde esta misma perspectiva, la infra-educación es prácticamente inexistente mientras que la sobre-educación afecta principalmente a los universitarios (58\%). Otro resultado es que los técnicos parecen estar mejor ubicados en el mercado laboral con solo un $10 \%$ caracterizado por sobrecualificación. Sin embargo, dentro de la encuesta del Ministerio de Educación, el 52\% de los egresados de carreras técnicas consideraron encontrarse en este tipo de desajuste. Asimismo, se encontró que los egresados universitarios son los que mejor están colocados en el mercado laboral con el $74 \%$ correctamente calificados. 


\section{GRÁFICO 1 \\ Comparación del desajuste educativo por niveles educativos \\ y métodos de cálculo}

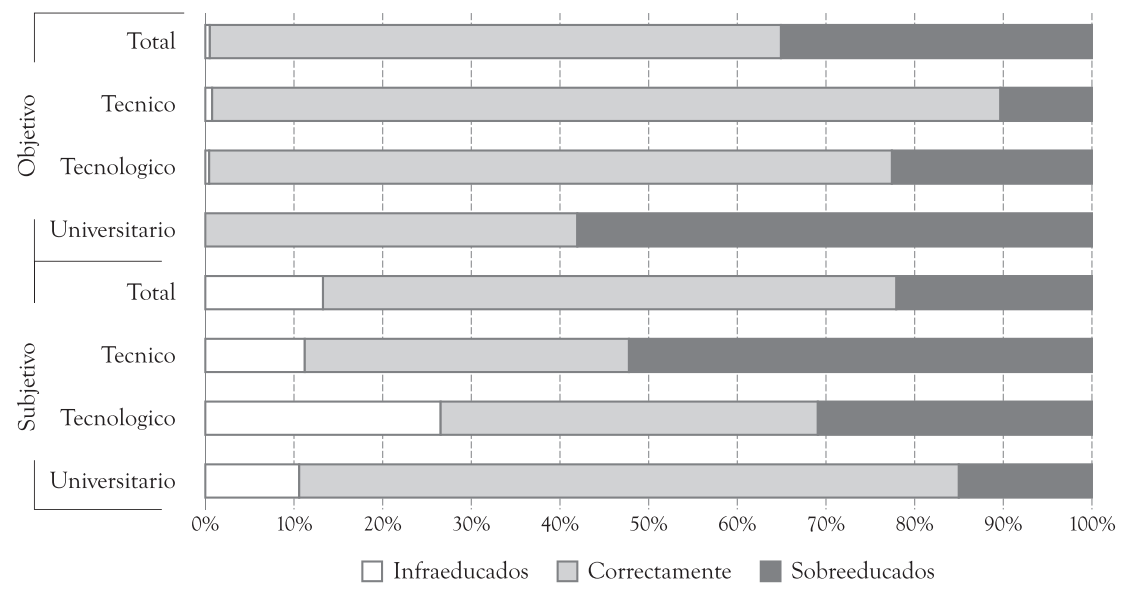

Fuente: cálculos de los autores con base en la ELPS y la Encuesta de egresados del Ministerio de Educación.

Habiendo calculado los niveles de desajustes, se estimó el modelo propuesto con el objetivo de conocer cómo afecta la situación de estar sobre/infra educado a los salarios. Después de depurar los datos eliminando las observaciones de individuos con datos incompletos, se examinaron los salarios de cerca de 1,3 millones de trabajadores. Los resultados se muestran en el Cuadro 3. En primera instancia se verifica la eficiencia del modelo, siendo el porcentaje de varianza captado por el modelo (R2) del 15\%. Segundo, se aprecia que el modelo en su conjunto es significativo porque se rechaza la hipótesis nula de no significancia mediante la lectura del chi cuadrado y su probabilidad.

Dentro del cuadro 3, los coeficientes de las variables categóricas señalan el cambio porcentual relativo del ingreso laboral de la persona en relación a una característica base. Por ejemplo, los hombres ganan un $9 \%$ más que las mujeres. Ahora bien, comprobando las hipótesis que atañen al desajuste educativo, se muestra que los egresados de educación terciaria que están en un primer empleo y que se han ubicado correctamente sus cualificaciones con sus competencias, ganan en promedio $29 \%$ más que las personas que se encuentran infra-educadas. 


\section{CUADRO 3}

Resultados de las estimaciones de la ecuación de Mincer

\begin{tabular}{|c|c|c|c|c|c|}
\hline Variable & $\begin{array}{l}\text { Característica } \\
\text { evaluada }\end{array}$ & Coeficiente & Error & Significancia & $\begin{array}{c}\text { Característica } \\
\text { base }\end{array}$ \\
\hline \multirow{2}{*}{ Desajuste } & Correctamente & 0,29 & 0,01 & 0 & \multirow{2}{*}{ Infraeducados } \\
\hline & Sobreeducado & 0,25 & 0,01 & 0 & \\
\hline Sexo & Hombre & 0,09 & 0 & 0 & Mujer \\
\hline Ocupación & Obrero Privado & $-0,09$ & 0 & 0 & $\begin{array}{c}\text { Obrero } \\
\text { Gobierno }\end{array}$ \\
\hline Sindicato & No & $-0,3$ & 0 & 0 & $\mathrm{Si}$ \\
\hline \multicolumn{2}{|c|}{ Constante } & 6,22 & 0,02 & 0 & \\
\hline \multicolumn{6}{|c|}{ Variables adicionales: departamento, rama de actividad, tamaño de la empresa, parentesco. } \\
\hline \multicolumn{3}{|c|}{ Observaciones $=1,372,683$} & \multicolumn{3}{|c|}{$F(90,1372583)=1589436$} \\
\hline \multicolumn{3}{|c|}{ Prob $>F=0.000$} & \multicolumn{3}{|c|}{ R-squared $=0.154$} \\
\hline
\end{tabular}

Fuente: cálculos de los autores con base en la ELPS y la Encuesta de egresados del Ministerio de Educación.

Otro resultado es que el ingreso de las personas que se encuentran sobre cualificadas es $25 \%$ mayor que el de las personas del grupo de referencia y, en consecuencia, los egresados correctamente cualificados en su empleo ganan $4 \%$ por encima que los que están sobre-educados. Este resultado es producto de que los empleos a los que acceden los sobre-educados, requieren una menor concentración de capital humano que se deriva en un menor empleo (Mincer, 1974; Ng, 2001). Este hallazgo es consistente con lo encontrado en la literatura internacional en lo referente al primer empleo (Velasco, 2004; López, 2008).

En otros resultados relevantes del modelo se aprecia que los egresados que laboran en el gobierno ganan un $9 \%$ más que los del sector privado, resultados que va en línea con la literatura científica reciente (De Castro, Salto y Steiner, 2013). Igualmente, los empleados inscritos en un sindicato poseen un ingreso de un 30\% por encima de los que no pertenecen, causado principalmente por el poder de negociación que pueden ejercer dentro de las empresas (Yamaguchi, 2016). 


\section{CONCLUSIONES}

El desajuste educativo es un fenómeno que afecta las inversiones en capital humano de los trabajadores, al desestimularlos por la expectativa de menores ingresos futuros. De ahí surge la importancia de estudiar la pertinencia de los planes de estudio y las competencias en el trabajo. En este sentido, este trabajo evidenció la presencia del desajuste educativo en el mercado laboral colombiano, y la metodología implementada mostró que el fenómeno es menos intenso del que se puede calcular mediante los métodos objetivos. En el primero se detectó una tasa del 35\% frente al segundo que fue del $46 \%$. En ambos métodos se aprecia que la sobre-educación es mayoritaria dentro de la población egresada; mientras que la infra- educación es menor o inexistente, lo que probablemente obedece a que los egresados universitarios consiguen trabajos, en general, para los que su nivel educativo se encuentra demasiado alto.

Asimismo, la sobre cualificación afectan más a los universitarios y a los técnicos. La teoría explica que este tipo de empleados aceptan estas vacantes porque están buscando la experiencia necesaria para poder aspirar a trabajos más especializados. No obstante, la falta de información longitudinal no permite confirmar esta hipótesis en periodos de tiempo más largos. Sobre este tema, la existencia de sobre-educación en todos los niveles educativos terciarios pone sobre la mesa el desfase entre las competencias que se adquieren en las instituciones educativas y las competencias que son necesarias en el mercado laboral.

En términos de salarios, se confirmó la hipótesis que evaluaba la diferencia salarial entre los correctamente y sobre cualificados, ganando estos últimos un $4 \%$ menos que sus pares. Este desfase sugeriría que los empleos en los que laboran tienen una menor necesidad de capital humano y por ende reportan menor remuneración. El modelo también muestra que los trabajadores pertenecientes a un sindicato y al sector público tienen mayores retornos salariales que sus contrapartes, lo que probablemente ocurre por el mayor poder de negociación que pueden ejercer en las convenciones colectivas. La ausencia de una base longitudinal también lleva a pensar que las diferencias en los coeficientes del desajuste educativo puedan reflejar los resultados de características no observables de las personas y no sólo la pérdida monetaria derivada del desajuste.

Esta diferencia amerita a futuro un estudio al detalle de las competencias exigidas a la fuerza de trabajo y que son enseñadas por los sistemas educativas, con el objetivo de hacerlos cada vez más pertinentes. Por tal razón los observatorios 
laborales para egresados son una herramienta que cobra utilidad para monitorear las tendencias en las competencias que necesitan los empresarios y así poder orientar las políticas de contratación. Finalmente, se requiere la construcción de mejores bases de información dado que no se poseen las necesidades implícitas de las empresas, los estudios suelen considerarlas de manera indirecta.

\section{REFERENCIAS}

Botello, Héctor (2015), "Evolución de las causas y efectos del desajuste educativo en el mercado laboral mexicano 1990-2010", Revista Económicas CUC, 36(1).

Carnevale, Anthony (1990), Workplace Basics: The Essential Skills Employers Want. ASTD Best Practices Series: Training for a Changing Work Force. San Francisco, CA: Jossey-Bass Inc., Publishers.

Castillo, Maribel (2007), "Desajuste educativo por regiones en Colombia: ¿competencia por salarios o por puestos de trabajo?", Cuadernos de Economía 26, 46.

Chávez, Álvaro y Arias, Helmuth (2002), Cálculo de la tasa interna de retorno de la educación en Colombia (Documento de Trabajo No. 2077). Bogotá: Universidad Externado de Colombia.

Cohn, Elchanan y Khan, Shahina (1995), "The Wage Effects of Overschooling Revisited", Labor Economics 2, 1.

De Castro, Francisco., Salto, Matteo y Steiner, Hugo (2013), The gap between public and private wages: new evidence for the EU (No. 508). Directorate General Economic and Financial Affairs (DG ECFIN), European Commission.

De Oliveira, Orlandina y Ariza, Marina (2000), "Género, trabajo y exclusión social en Colombia", Estudios demográficos y urbanos, 11-33.

Dorn, David y Souza-Poza, Alfonso (2005), "Overqualification: Permanent or Transitory", conferencia presentada en la International Conference on Educational Economics, Estonia, University of Tartu, 26-28 de agosto de 2005.

Flisi, Sara., Goglio, Valentina., Meroni, Elena., Rodrigues, Margarida., y Vera-Toscano, Esperanza (2017), "Measuring occupational mismatch: overeducation and overskill in Europe-Evidence from PIAAC", Social Indicators Research, Vol. 131, No. 3.

Freeman, Richard (1975), The Overeducated American, New York: Academic Press.

Frei, Christa y Sousa-Poza, Alfonso (2012), "Overqualification: permanent or transitory?", Applied Economics Vol. 44, No. 14. 
Groot, W., y H. van den Brink (2000), "Overeducation in the Labor Market: A Meta Analysis", Economics of Education Review 19, 2.

Hartog, Joop (2000), "Over-education and Earnings: Where are We, Where Should We Go?", Economics of Education Review, 19.

Johnson, William (1978), "A Theory of Job Shopping", Quarterly Journal of Economics 92.

Kiker, Billy. F.; Santos, Maria C. y M. Mendez. de Oliveira (1997), "Overeducation and Undereducation: Evidence for Portugal", Economics of Education Review 16.

López, Martha. R (2008), "Un análisis del desajuste educativo en el primer empleo de los jóvenes", Principios: Estudios de Economía Politica, 11.

McGuiness, Seamus (2006), "Overeducation in the Labour Market", Journal of Economic Surveys 20, 3.

Mincer, Jacob (1974), "Schooling, Experience, and Earnings", Human Behavior Eु Social Institutions No. 2.

Mora, Jhon James (2004), "Sobre-educación en Cali (Colombia), ¿Desequilibrio temporal o permanente?: Algunas ideas, 2000-2003", Revista de Economía y Administración 1.

Mora, Jhon James y Muro, Juan (2008), "Sheepskin Effects by Cohorts in Colombia", International Journal of Manpower 29, 2.

Ying Chu Ng (2001), "Overeducation and Undereducation and their Effect on Earnings: Evidence from Hong Kong, 1986-1996", Pacific Economic Review 6.

Rosen, Sherwin (1972), "Learning and Experience in the Labour Market", Journal of Human Resources 7.

Rubb, Stephen y Quinn, Michael (2002), Educational Mismatches in Colombia: Additional Evidence of the Importance of Labor Market Assignments?, artículo presentado en la International Atlantic Economic Society Meeting, Washington.

Sicherman, Nachum (1991), "Overeducation in the Labor Market", Journal of Labor Economics 9, 2.

Velasco, Manuel (2004), "La relación educación-economía: un estudio del desajuste educativo de los titulados universitarios", Revista de Educación, 334.

Verdugo, Richard y Verdugo, Naomi (1989), "The Impact of Surplus Schooling on Earnings", Journal of Human Resources 24.

Yamaguchi, Shintaro (2016), "Changes in returns to task-specific skills and gender wage gap", Journal of Human Resources, 53. 